\title{
« Ma situation était un peu comparable à celle des scientifiques... »
}

Un entretien avec le compositeur Hanspeter Kyburz sur son œuvre The Voynich Cipher Manuscript (1995)

\section{Pierre Michel}

\section{CpenEdition}

\section{Journals}

Édition électronique

URL : http://journals.openedition.org/rbnu/1523

DOI : $10.4000 /$ rbnu. 1523

ISSN : 2679-6104

Éditeur

Bibliothèque nationale et universitaire de Strasbourg

\section{Édition imprimée}

Date de publication : 1 mai 2016

Pagination : $72-79$

ISBN : 9782859230623

ISSN : 2109-2761

\section{Référence électronique}

Pierre Michel, « « Ma situation était un peu comparable à celle des scientifiques... » », La Revue de la BNU [En ligne], 13 | 2016, mis en ligne le 01 mars 2020, consulté le 21 décembre 2020. URL : http:// journals.openedition.org/rbnu/1523; DOI : https://doi.org/10.4000/rbnu.1523

\section{(c) (i) (2)(2)}

La Revue de la BNU est mise à disposition selon les termes de la Licence Creative Commons Attribution - Pas d'Utilisation Commerciale - Partage dans les Mêmes Conditions 4.0 International. 


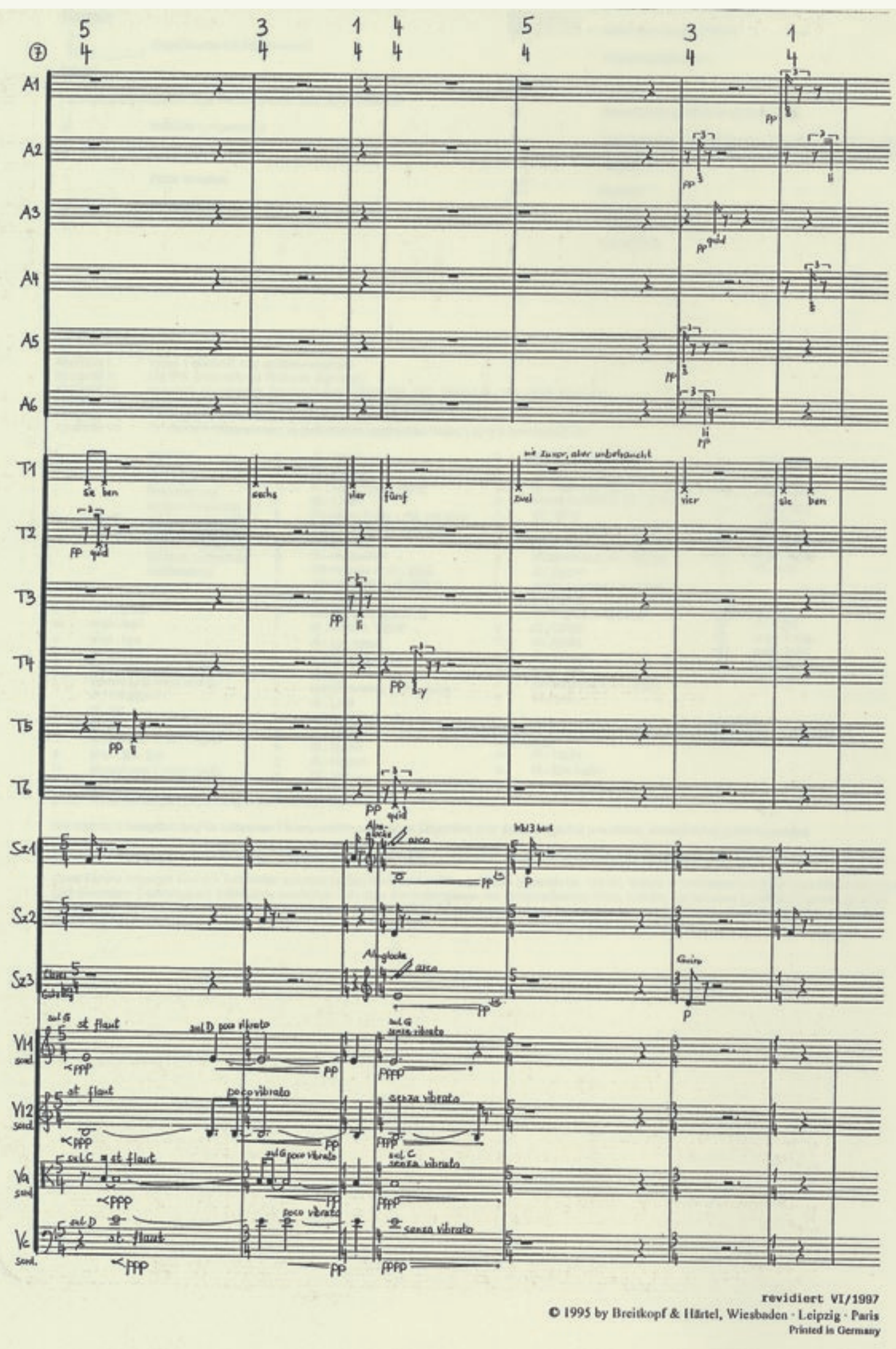

Extrait de la partition (1̀re partie), montrant en particulier les chiffres énumérés par le ténor. La partition a été éditée par Breitkopf \& Härtel (Wiesbaden, 1997 ; coll. BNU). 


\section{« MA SITUATION ÉTAIT UN PEU COMPARABLE À GELLE DES SCIENTIFIQUES... 》 Un entretien avec le compositeur Hanspeter Kyburz sur son œuvre The Voynich Cipher Manuscript (I995)}

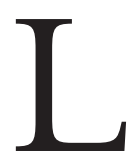

e manuscrit de Voynich a fasciné bien au-delà du monde des bibliothèques ou de la littérature au sens large du terme. Pour ce dossier de La Revue de la BNU, nous avons pu réaliser un entretien avec le compositeur Hanspeter Kyburz autour d'une de ses œuvres, The Voynich Cipher Manuscript, qui se penchait en 1995 sur ce manuscrit de façon très personnelle. Hanspeter Kyburz est un compositeur suisse né en 1960 au Nigeria, et qui vit en Allemagne depuis l'âge de dix ans. Il a fait des études de composition d'abord à Graz avec Gösta Neuwirth, puis à Berlin en 1982 où il a étudié, outre la composition avec Frank Michael Beyer et Gösta Neuwirth, la musicologie, l'histoire de l'art et la philosophie jusqu'en 1991. Après s'être perfectionné auprès de Hans Zender à Francfort, il a reçu le Prix Boris Blacher en 1990, le Prix SchneiderSchott en 1994, le prix d'encouragement de l'Académie des Arts de Berlin en 1996 et le prix de la Fondation Ernst von Siemens en 2000. Il a enseigné dans différents studios d'électroacoustique en Allemagne, en Autriche et en Suisse avant d'être nommé en 1997 professeur de composition à la Hochschule für Musik Hanns Eisler de Berlin. En 1998, Kyburz fut également chargé de cours à l'Académie d'été de Darmstadt, et de 2000 à 2002, à la Musikhochschule de Bâle où il dirigea aussi le Studio de musique électronique.

Ce compositeur fut programmé à plusieurs reprises au Festival Musica, et The Voynich Cipher Manuscript fut joué le 27 septembre 2003 à Strasbourg (Palais de la Musique et des Congrès) dans le cadre du festival. Le compositeur était également l'invité de Philippe Manoury lors de son académie de composition à Strasbourg en 2015, et il participa, à l'occasion de l'édition 2015 de Musica, à un entretien public à la BNU le 30 septembre 2015.

\section{Comment se situe The Voynich Cipher Manuscript dans votre parcours de compositeur?}

C'est difficile à dire. Au début, après mes études, j’ai commencé avec les algorithmes. D’abord avec une pièce pour soliste et ensemble (Cells), puis une pièce pour grand ensemble (Parts), puis celle-ci qui était ma troisième pièce algorithmique, utilisant la voix pour rechercher des relations entre des processus algorithmiques et quelque chose qui ressemble à une langue. Qui n'en est pas vraiment une, mais qui... C'est plutôt une imprévisibilité des éléments algorithmiques comparée à une imprévisibilité phonétique d'une langue. Mais quand même, on a des couches sémantiques, des structures de phrases, plutôt qu'une suite de composantes avec du sens. C'est donné par le caractère de la musique, c'est plutôt le geste, l'instrumentation, les couleurs qui nous font comprendre qu'il s'agit de quelque chose de non connu, mais dont on peut estimer un peu le caractère.

\section{Il semble que dans plusieurs de vos œuvres vous ayez été intéressé par des algorithmes, par les " possibilités de connexion d'éléments plus ou} moins complexes et hétérogènes "... Par le L-System (du biologiste Lindenmayer) ${ }^{1}$. Cela se situait donc avant The Voynich Cipher Manuscript ?

Je n'ai pas tout de suite connu Lindenmayer. Je cherchais quelque chose au-delà d'une polarité, qui m'a toujours un peu poursuivi, c'était la stabilité ou la continuité des "patterns " stables chez Ligeti (ses processus étaient tout à fait prévisibles, surtout dans sa musique des années 1960). À l'inverse, on a eu des moments isolés de Lachenmann, quelque chose qui sonne de façon très intéressante, mais où l'on ne peut pas prévoir ce qui se passe. Cette polarité m'a guidé un peu

(suite p. 76) 
$17+4 \quad 5000$ Ig saw g g reor sam of reane

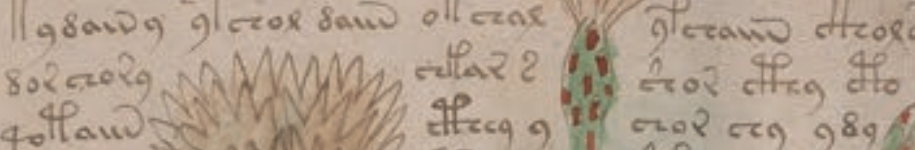

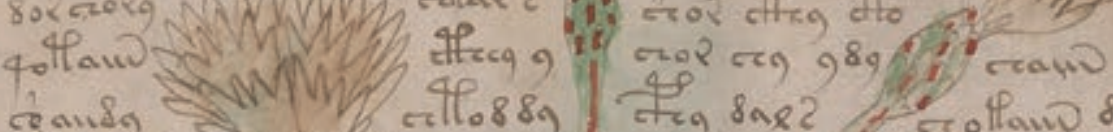

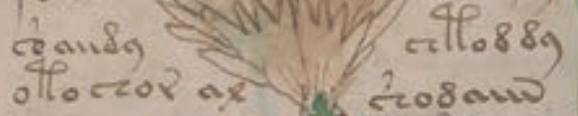
zam 8aws of 8thor 202 trox effeq sawo of coos 800

\begin{tabular}{ll}
-008 & 800 \\
\hline
\end{tabular} Erotham 8 woplyys

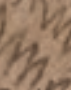

cos 8 no glterano sino

9lior8y secos 8cz Ift?

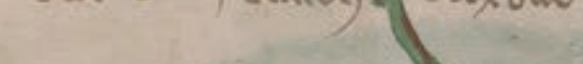

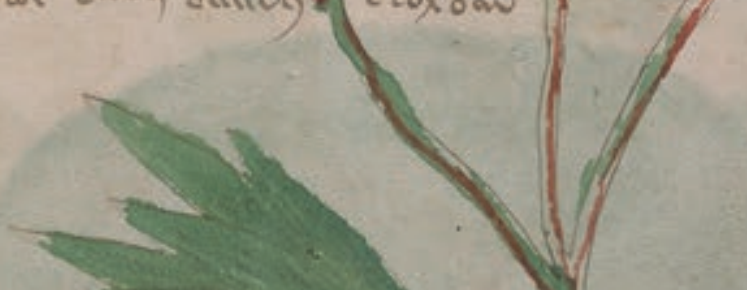

lgsaro żano 4092 coos Posno 802 es. lllor efera folleq gillosa zo

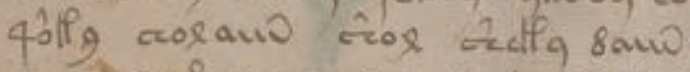
gezaro salezg saroz ćeal sao Plos ry col Afrov goर al cal fillaio eca 8 amp

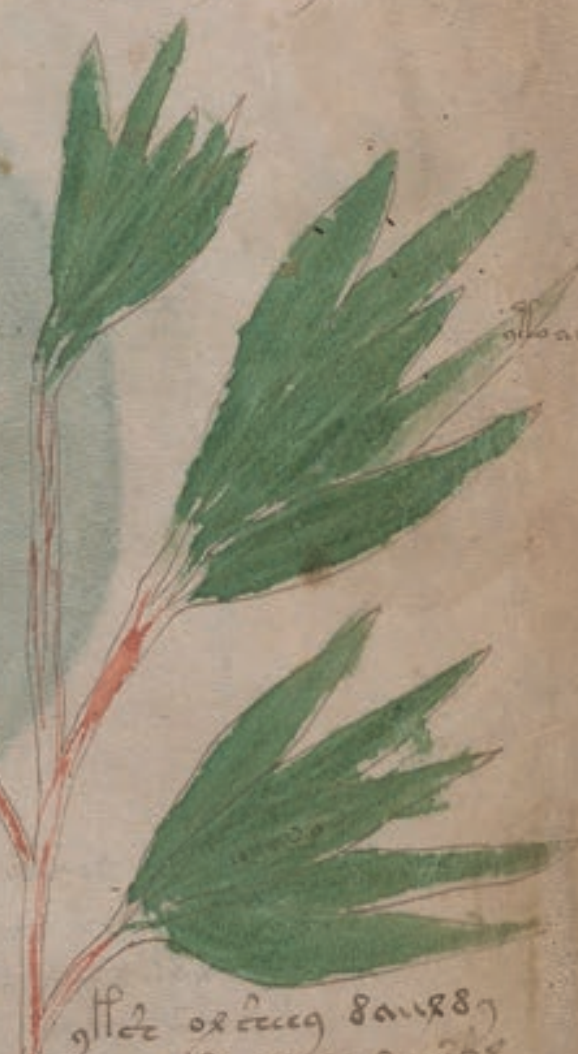
?ीटि of zecen 8 ants 8 ? zoxatla savo sanisos iffecg Iflas zawo zan oxरan ciceg df co?

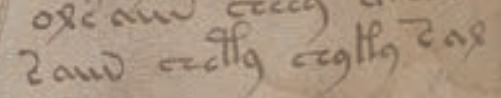

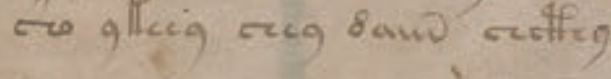

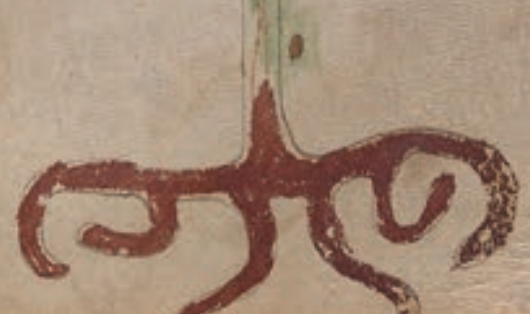




\section{Le " manuscrit Voynich", le texte le plus mystérieux du monde?}

Son nom. En 1912, un marchand de livres anciens, polonais d'origine, Wilfrid Voynich, achète à Rome à des jésuites, dans le plus grand secret, un choix d'imprimés et de manuscrits. L'un d'entre eux retient d'emblée son attention : il est rédigé dans un alphabet et une langue inconnus de lui. Il y consacrera sa vie. On parle donc du « manuscrit Voynich ».

Son aspect. De taille modeste $(22,5 \operatorname{sur} 16 \mathrm{~cm})$, couvert d'un vélin sans titre et sans nom d'auteur, constitué de feuillets inégaux, il est de facture commune et partiellement endommagé. La grande majorité des folios est illustrée : plantes de fantaisie, croquis cosmologiques, figures du zodiaque et foisonnement d'énigmatiques créatures féminines qui partagent l'espace avec une écriture plutôt soignée, à l'encre brune, indéchiffrable.

Son lieu de conservation. Voynich a joué un rôle déterminant dans la valorisation du manuscrit, mû à la fois par une curiosité scientifique réelle et le désir d'en augmenter la valeur marchande. Cultivé, doué d'une imagination fertile et sachant s'entourer d'intellectuels de qualité, il ouvre une véritable " enquête " et met tout en œuvre pour lever le mystère, s'impliquant lui-même dans cette quête et sollicitant des scientifiques de tout type. Il poursuit ses recherches aux États-Unis où il s'installe dès 1915. Il garde dans ses collections, jusqu'à sa mort, le précieux manuscrit qui porte son nom. Sa secrétaire le revend quelques années plus tard à un marchand qui, à son tour, faute d'acquéreur en raison de son prix, en fait don à la Beinecke Rare Book \& Manuscript Library de l'Université de Yale. Il s'y trouve actuellement sous la cote MS 408.

Son auteur et son contenu. On doit les premiers postulats à Voynich en personne. S'aidant d'une lettre en latin jointe au manuscrit et qui suggère clairement cette hypothèse, il attribue ce texte - qu'il identifie comme " codé " - au moine franciscain érudit du $13^{\mathrm{e}}$ siècle Roger Bacon. L'homme qui tenta secrètement de réformer la scolastique au profit des sciences expérimentales et se passionna pour la médecine, l'astronomie mais aussi l'astrologie et les mystères de l'alchimie lui paraît correspondre aux hypothèses que l'on peut faire au regard des illustrations. Poursuivant son enquête sur les éventuels possesseurs du manuscrit et sur sa " traçabilité ", il tombe toujours sur des passionnés d'alchimie et d'ésotérisme, retrouvant trace d'une arrivée à prix d'or, à la fin du $16^{\mathrm{e}}$ siècle, à la cour de Prague, entre les mains du roi Rodolphe II, autre féru des sciences occultes. L'hypothèse d'un texte crypté va de soi pour de semblables sujets... mais l'alphabet et le code demeurent une énigme pour les plus savants les plus avertis du temps, qui y vouent pourtant « un labeur infatigable ", comme le rapporte la lettre datée de 1666 et jointe au manuscrit.

\section{Un défi aux amateurs comme aux spécialistes de} la cryptographie. Sa vie durant, Voynich sollicite des spécialistes de tout poil : philosophes, juristes, paléographes, linguistes, médecins et scientifiques relèvent le défi sans résultats probants. Les hypothèses linguistiques les plus fantaisistes et les plus opposées sont envisagées sans jamais donner de résultats définitifs. Le colonel américain Friedman, le spécialiste de cryptographie qui avait fait merveille pour " casser » les codes japonais durant la Seconde Guerre mondiale, passionné par le manuscrit depuis les années 20, échoue à son tour en 1944. Après une dernière tentative, il conclut à un langage inventé de toutes pièces... L'hypothèse d'une imposture ou d'un canular ne décourage toutefois pas les recherches qui, jusqu'à aujourd'hui, se poursuivent et mettent en œuvre les ressources les plus performantes.

Accessibilité accrue. Un chercheur de l'Université d'Arizona a pu,en 2011, grâce au carbone 14, affirmer que le vélin du manuscrit avait été fabriqué entre 1404 et 1438 : c'en fut fait de l'hypothèse de Roger Bacon comme auteur... Le manuscrit reste à la disposition des spécialistes à Yale, qui en a mis en ligne une version numérisée ${ }^{1}$, mais des éditions diverses ${ }^{2}$ l'ont d'autre part rendu accessible en fac-similé (voir un exemple page ci-jointe), et à défaut de le comprendre on peut en admirer les illustrations qui ne ressemblent, de fait, à rien de connu... et sont de nature à solliciter bien des imaginaires, en musique comme en d'autres domaines.

\footnotetext{
1 - http://brbl-dl.library.yale.edu/vufind/Record/3519597

2 - Voir en particulier, en français, Le Code Voynich. Le manuscrit MS 408 de la Beinecke Rare Book and Manuscript Library Yale University, texte d'introduction de Pierre Barthélémy, Paris, Jean-Claude Gawsewitch, 2005
} 
dans la recherche de mes propres moyens pour contrôler la périodicité, autrement dit l'équilibre entre quelque chose de complètement continu et l'imprévisibilité totale d'éléments stochastiques. Je voulais trouver quelque chose entre les deux, mais pas en interpolant des éléments de l'extérieur (par exemple 1, 2, 3 dans n'importe quelle position, puis on intègre le 4, puis le 5), plutôt en générant une sorte d'instabilité de l'intérieur en cherchant des règles qui me permettent de contrôler cela. Au moment où j'ai vu des formules comme " $\mathrm{L}(\mathrm{L})$ ", ça m'a donné des idées et ouvert des possibilités que je ne connaissais pas, et j'ai tout de suite commencé à programmer ${ }^{2}$. Après, j'ai découvert Lindenmayer et ses systèmes de "re-write" : ils ont remplacé toute une séquence de symboles par d'autres symboles, par d'autres séquences. De cette façon les " mots" pour les mathématiciens sont remplacés par des mots. Cela suggère quelque chose comme une langue ; si l'on veut, on peut en déduire toute une grammaire générative avec n'importe quel nombre de nouveaux éléments. Cela m'a fasciné, mais il m'a fallu quand même à peu près trois ans, même plus, pour préciser les objets musicaux qui sont intéressants avec ce parti-pris. J'ai commencé avec des rythmes, des hauteurs, ensuite plutôt avec des motifs, d'une mesure ou de plusieurs mesures.

\section{Comment en êtes-vous venu à ce " manuscrit de Voynich " (qui fut découvert en 1912 dans un collège jésuite près de Rome) ?}

Je n'y suis pas arrivé par hasard, c'est par mon épouse : elle connaissait l'existence du manuscrit par Umberto Eco, car il l'avait déjà décrit. Ma femme m'a en effet montré un article d'Eco qui mentionnait ce manuscrit. Il n'était pas au centre de l'article, mais j'ai quand même commencé à chercher du matériau... ${ }^{3}$ On était au milieu des années 1990, il n'existait que deux ou trois livres, c'était presque impossible. La documentation n'était pas aussi développée qu'aujourd'hui ; actuellement le manuscrit est beaucoup plus connu, de nombreux scientifiques s'en occupent. Et la technique de la traduction analytique s'est beaucoup développée car les linguistes maintenant ont affiné leurs méthodes. À l'époque, c'était un peu " historique " : beaucoup de recherches tentaient de trouver le code, mais après un certain temps le manuscrit était de nouveau une énigme. Le code était toujours faux, mais quand même : les chercheurs les plus reconnus étaient absolument convaincus qu'ils allaient le trouver. Mais trois mois plus tard, quelqu'un d'autre trouvait un autre code, et ainsi de suite (rires). Ce processus de recherche m’a beaucoup plus intéressé que le sens définitif du ma- nuscrit, parce qu'il produisait beaucoup de matériau. On sait que c'est le texte d'un apothicaire, c'est plutôt de l'alchimie : il s'agit de la façon dont générer de l'or, donc c'est une bataille décrite de façon systématique (non pas historique), les spéculations sont infinies. Donc c'était un peu cela : j'imaginais un scientifique travaillant à sa table, et... Que fait-il ? Il compte les syllabes, les chiffres, n'importe quoi, les illustrations, il se parle et il commence à compter, c'est l'action du ténor de la première section de mon œuvre.

\section{Donc celui qui énumère les chiffres. Dans votre pièce, on observe cela à plusieurs moments. Dans la première partie, des chiffres sont dits en allemand (voir ill. p. 78) : 21624176 , puis dans la troisième partie on retrouve ces chiffres (p. 28 de la partition) 24 , puis, p. 31, 94857 , etc. On retrouve ceci aussi à la fin de l'œuvre (p. 84) : 12514 2, etc. Est-ce en rapport direct avec la musique?}

Oui, absolument. Les scientifiques travaillent avec des symboles, mais pour des raisons pratiques j'ai utilisé des chiffres. J'aurais pu utiliser l'alphabet, ou n'importe quoi d'autre. Avec les chiffres je commence à numéroter les éléments musicaux : premier moment, deuxième moment, etc. C'est un peu la chronologie du développement musical, même si je sais qu'il ne s'agit pas du tout d'un développement linéaire, mais quand même l'inscription des éléments, la chronologie de l'introduction me donnent une raison pour désigner les choses : ceci est le 1, ceci est le 2, etc. Ensuite, la séquence parlée du ténor est dérivée de cette séquence originelle, simplement pour indiquer un peu la relation indirecte entre un symbole et sa signification. Il dit " 2 », mais pour moi, sur le plan du compositeur, c'est « 1 ». Ainsi le ténor identifie quelques éléments musicaux qui l'entourent.

\section{Ces suites de chiffres sont-elles empruntées à une partie du manuscrit ?}

Non, ces chiffres sont un moyen pour théâtraliser le processus d'un scientifique qui travaille sur le manuscrit. Le ténor est plus ou moins un alter ego pour moi, quelqu'un qui me fait rentrer dans la science du manuscrit. Mais il reste une personnalité inventée : alors tout ce qu'il dit n'est pas vraiment rationnel, et de plus en plus il est porté par le développement musical, il n'est plus autonome, il ne garde pas sa distance envers l'objet du manuscrit. De plus, il est porté par ses spéculations, et aussi naturellement par le développement de la musique. 


\section{C'est ce que vous appelez « la dynamique complexe de l'acte traducteur ". Si je comprends bien, le manuscrit vous a inspiré dans tout ce qu'il impliquait pour les chercheurs.}

Oui, parce que ces gens ont produit des textes, des traductions, parfois même des chapitres, parfois simplement quelques phrases, mais j'ai été impressionné par l'activité même des chercheurs. Peut-être devrait-on mentionner aussi les Américains qui ont décodé pendant la Seconde Guerre mondiale le code des Japonais. Mais ces gens dont je parlais ont commencé avec un projet scientifique à l'Université de San Francisco, avec beaucoup d'argent, et naturellement on a trouvé un code, ou plusieurs codes, et on a produit des textes, des matériaux, et à la fin tout était faux (rires). Et ça m'a toujours fait plaisir que l'intelligence analytique ne suffise pas ; c'est surtout le contexte pragmatique qui reste à découvrir, parce que sans ça on ne sait pas comment les gens ont utilisé ce manuscrit, dans quel contexte il était considéré comme important. Par exemple, on dit que l'empereur Rudolf II aurait payé le prix incroyable de 600 ducats (aujourd'hui on pourrait acheter toute l'Autriche avec l'équivalent), donc il a fait n'importe quoi simplement pour l'avoir, sans pouvoir le lire. C'est presque ridicule ! Et cela m’a toujours fasciné, ce côté analytique parfois pauvre et très têtu, parfois spéculatif et riche sans fondement analytique... Cette tradition, ces développements, c'est un peu comparable à mes études de jeune compositeur qui voulait trouver une base, qui avait beaucoup de moyens techniques pour arriver à quelque chose musicalement, mais sans fondement théorique ; allant dans plusieurs directions mais sans avoir... par exemple les relations mutuelles entre l'harmonie et le rythme... Tout cela était très spéculatif. Et de ce point de vue ma situation était un peu comparable à celle des scientifiques.

\section{Le chœur chante tantôt des onomatopées ou des mots issus du manuscrit, tantôt (à trois reprises) des poèmes de Chlebnikov (selon la traduction allemande d'Oskar Pastior). Qu'est-ce qui justifie cet ajout ?}

J'ai ajouté des poèmes de Chlebnikov dans la traduction d'Oskar Pastior, qui est un auteur proche pour moi. J'ai écrit des poèmes pour son soixantième anniversaire. Mais je ne le connaissais pas avant d'avoir lu ses traductions de Chlebnikov. Ces textes sont pleins de néologismes, c'est une langue constructive, mais aussi déconstructive, parce qu'il a pareillement recherché les racines des mots, leur étymologie, et sur cette base il a commencé à construire quelque chose d'inconnu. Pastior n'a pas seulement traduit le texte, mais aussi la façon de travailler ; alors il a cherché des analogies allemandes, il a travaillé comme Chlebnikov, et c'est pour cela que les textes sont impressionnants, car il ne s'agit pas seulement de la personnalité artistique de Chlebnikov ou de Pastior, mais aussi de l'activité traductrice en soi. C'est quelque chose de tout à fait construit, mais avec une profondeur étrange ; les textes sont très similaires, mais il est difficile d'expliquer pourquoi.

\section{Il faudrait suivre le processus de tout cela...}

Oui, il faudrait le refaire. Et lorsque j'ai composé avec ces textes, il m'a fallu remonter le processus, et cela fut une véritable découverte de textes que je n'avais pas lus auparavant. Je découvrais donc un texte en faisant la musique. Souvent, lorsqu'on écrit une œuvre, on découvre certains aspects du texte, mais lorsqu'il s'agit d'une langue artificielle, cela change beaucoup, on n'est plus tellement auto-confident : comment doit-on faire chanter un mot comme " schtorb "... ? C'est " mourir ", pas vraiment, mais un peu... C'est tout l'entourage du champ sémantique d'un mot.

\section{J’ai remarqué que dans les différentes parties de l'œuvre il y a des textes, par exemple dans la partie $V$. Ces mots ne sont pas issus des poèmes de Chlebnikov; d'où viennent-ils ?}

Du manuscrit ! Des traductions du manuscrit. Ce sont des traducteurs qui ont trouvé cela. C'est un texte de pharmacien, cela fait partie de tout un univers d'hypothèses.

\section{Et dans d'autres parties (comme dans la première partie), il y a des onomatopées...}

Non, ces sont les consonnes qui produisent des mots, par exemple " $s$ " pour " salt». Ce sont des nuages de consonnes (entre les différentes voix) qui entourent une pensée scientifique à la recherche du sens (le ténor).

\section{Comment est venue l'idée de mettre ces textes de Chlebnikov dans l'œuvre ? C'était au moment où vous avez réfléchi au manuscrit ?}

Non, l'explication est très simple. J'avais eu des matériaux des traductions ou pseudo-traductions du Voynich Cipher Manuscript et j'ai cherché un contraste, quelque chose de plus "reliable ", quelque chose qui me donne une forme à long terme, pas seulement pour cinq minutes, mais pour vingt minutes, et pour cela j'ai cherché un élément plus cohérent ; pas consistant, je ne 
cherchais pas un système rationnel, mais quelque chose ayant un caractère, comme par exemple " Feurott " 4, très spécifique par rapport aux autres sections, qui me donne la possibilité de construire la musique au-delà de trois minutes. Et cela n'aurait pas été possible sur la base des matériaux des traductions du manuscrit, car à long terme cela devient ennuyeux.

\section{C'est comme une structure seconde?}

Oui, cela portait les matériaux des traductions, car les chanteurs nous permettent de nous identifier à long terme avec un caractère.

\section{Il y a une spatialisation de la musique dans cette œuvre...}

Oui, autour du public il y a tous les instruments, trois percussionnistes et les chanteurs, et au milieu du public il y a quatre solistes : soprano, alto, ténor et basse.

\section{L'œuvre est en six parties, comment vous est venue cette conception?}

C'est très simple, car j'ai trois poèmes, et j'interpose les trois poèmes avec les matériaux des traductions du manuscrit : donc c'est une forme ABABAB.

\section{Connaissiez-vous d'autres musiciens qui avaient travaillé sur ce manuscrit ?}

Non, mais certains compositeurs souhaitent travailler selon le manuscrit, et sur Internet on trouve plusieurs compositions, qui ne sont pas forcément ambitieuses, mais qui s'inspirent du manuscrit. Pour moi, l'enjeu de l'ajout des poèmes était justement de m'en libérer un peu.

\section{Retrouve-t-on par la suite ce genre d'orientation chez vous?}

Non, c'était une étape, c'est pour ainsi dire un " autre " qui a écrit cette pièce, quelqu'un qui s'est posé ces questions, a rencontré ces problèmes, ces potentialités. Le contexte vocal avait déterminé cette œuvre, mais maintenant cela a beaucoup changé pour moi. Car j'ai besoin d'un nouveau contexte pour chaque pièce.

\section{Avez-vous un intérêt pour d'autres manuscrits étranges de ce genre?}

Une fois, lorsque j'étais avec Betty Freemann à New York, je lui ai dit que j'aimerais travailler sur les manuscrits de Beethoven, parce qu'ils sont très peu lisibles! Tout le monde connaît Beethoven, mais les manuscrits sont presque toujours illisibles ! Je lui ai dit que j'aimerais travailler sur le manuscrit de la
Hammerklavier. Et j'ai pu le consulter grâce à elle à la Morgan Library à New York. Beethoven a écrit plusieurs lettres à Czerny au sujet de la façon dont quitter la tonalité. Alors Czerny lui a dit : avec la fugue, mais Beethoven lui a répondu qu'il avait besoin d'une nouvelle poétique pour la polyphonie (l'écriture héritée de Bach). Et pourtant, après avoir abandonné, il a repris cette fugue de la Hammerklavier avec, dans le manuscrit, des altérations qui sont parfois étranges, qui ne sont plus tonales. C'est pourquoi j'ai lu précisément ce manuscrit pendant plusieurs jours. C'est évident qu'il a cherché une nouvelle écriture polyphonique dans cette fugue. Les motifs et le contrepoint changent de registre, avec des croisements ; il est très difficile de suivre les voix. La voix comme construction centrale de la polyphonie est perdue intentionnellement ; c'est vraiment au cœur de l'idée poétique de Beethoven. Il a cherché à nous " irriter ", il a cherché la confusion, parce qu'on dépendait beaucoup de la continuité du registre pour une voix. Dans le manuscrit, il a corrigé une centaine de fois la fugue, surtout les altérations. Et cela m'a beaucoup fasciné, c'était une expérience secondaire après le Voynich Cipher Manuscript. Cette idée d'échapper à la tonalité était une chose complètement folle pour l'époque !

\section{Propos recueillis par Pierre Michel}

(Strasbourg, le 28 septembre 2015)

\footnotetext{
Notes

1 - Un L-System ou système de Lindenmayer est une grammaire formelle, inventée en 1968 par le biologiste hongrois Aristid Lindenmayer, qui consiste à modéliser le processus de développement et de prolifération de plantes ou de bactéries.

2 - Cette formule brève m'a frappé comme un noyau qui indiquait en un instant tout le principe de la récursivité. Déployée, la formule décrit un processus génératif : une instance d'un objet nommé $\mathrm{L}$ sera remplacée par une autre instance du même objet, quelle que soit la complexité interne de cet objet.

3 - En particulier William Poundstone (Im Labyrinth des Denkens, Hambourg, 1995, p. 293 et suiv.) qui décrit le manuscrit et a ainsi attisé ma curiosité, et Umberto Eco (Die Suche nach der vollkommenen Sprache, Munich, 1994, p. 194 et suiv.) qui évoque, lui, la « langue magique » de John Dee.

4 - Ce mot débute le deuxième poème de Chlebnikov utilisé dans l'œuvre ( $4^{\mathrm{e}}$ partie).
}

Ecouter The Voynich Cipher Manuscript : un CD Kairos (Malstrom, The Voynich Cipher Manuscript, Parts), réf. $0012152 \mathrm{KAI}$

- En savoir plus sur Hanspeter Kyburz : http://brahms.ircam.fr/hanspeter-kyburz 


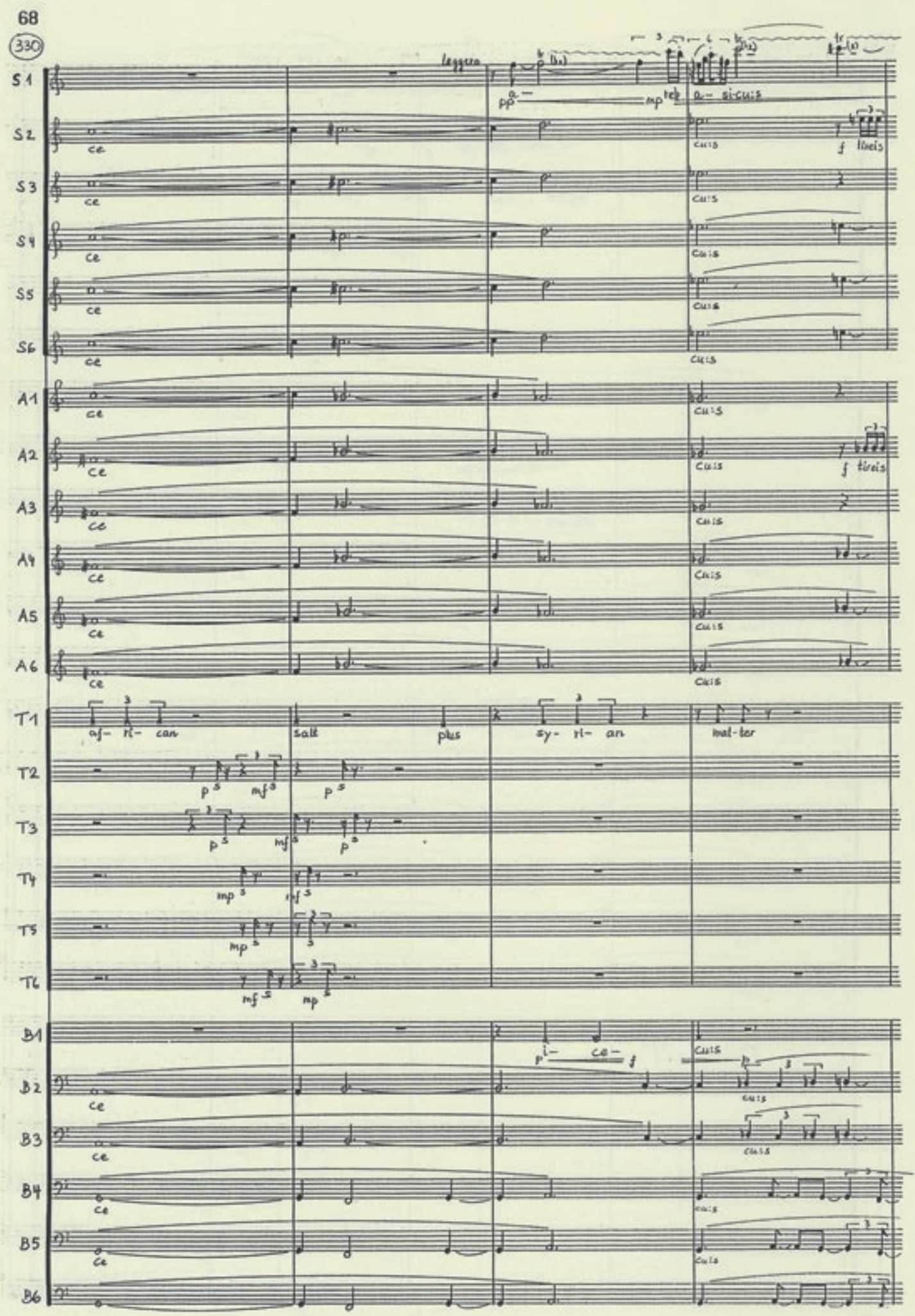

Extrait de la partition ( $5^{\mathrm{e}}$ partie),

avec l'apparition du mot « salt " (coll. BNU). 